Original Article

\title{
A kinematic analysis of the lower limb with regard to restricted spinal motion during gait
}

\author{
Hyeon-Nam Song, PhD, PT ${ }^{1)}$, Young Mi Kim, PhD, PT ${ }^{1)^{*}}$, Kyoung Kim, PhD, $\mathrm{PT}^{2}$ ) \\ 1) Department of Rehabilitation Science, Graduate School, Daegu University, Republic of Korea \\ 2) Department of Physical Therapy, College of Rehabilitation Science, Daegu University: \\ 201 Daegudae-ro, Jillyang, Gyeongsan-si, Kyeongbuk 712-714, Republic of Korea
}

\begin{abstract}
Purpose] The purpose of this study was to investigate the effect of restricted spinal motion on kinematic changes in the lower extremities using a rigid thoracolumbosacral orthosis. [Subjects and Methods] Forty healthy males in their 20 s were selected as the sample, which was randomly and evenly divided into two groups: (1) the WT group (with a thoracolumbosacral orthosis) and (2) the WOT group (without a thoracolumbosacral orthosis). The spinal orthosis used in this study was a thoracolumbosacral orthosis called a plastic body jacket. [Results] The sagittal plane; in the level ground walking measurements, significance differences were found at the H2 (Hip maximum flexion/extension in midstance phase) and K2 (Knee maximum flexion/extension in midstance phase) between the WT group and the WOT group. [Conclusion] It can be concluded that a spinal orthosis is useful in stabilizing the lower extremities during stair gaiting, and that appropriate application of the orthosis plays a supporting role in the activities of daily life and therapeutic intervention.

Key words: Kinematic analysis, Restricted spinal motion, Gait
\end{abstract}

(This article was submitted Jul. 28, 2016, and was accepted Oct. 5, 2016)

\section{INTRODUCTION}

The spine creates a closed kinematic chain through segmental configuration of the upper and lower extremities and the pelvic girdle, influencing static and dynamic balance during sports activities and the activities of daily life ${ }^{1,2)}$, while also having a direct effect on postural alignment through musculoskeletal connections ${ }^{3)}$. However, if a normal form cannot be maintained due to internal and external forces, any force applied to the spine can cause misalignment, causing lateral imbalance and the accumulation of fatigue in the adjacent muscle, which often results in chronic low back pain ${ }^{4}$.

Treatment with a rigid thoracolumbosacral orthosis is the most common non-operative treatment for the prevention of curve progression. There are many different brace designs, but with all of them, the objective is to restore the normal contours and alignment of the spine by means of external forces and, in some designs, the stimulation of active correction as the patient moves the spine away from pressures within the brace ${ }^{5}$. A thoracolumbosacral orthosis (TLSO), a brace that extends from the spine to the pelvis, has a direct impact on the movement of the spine during walking and hence has a significant influence on the movement of the pelvis and the lower limbs.

Spinal orthoses are commonly used in the treatment of various conditions that affect the spine. Therefore, in this study, we examined the impact on the pattern of walking on both level ground and stairs after restricting the movement of the spine and the trunk of the body through the use of a spinal orthosis.

\section{SUBJECTS AND METHODS}

In this study, 40 healthy males in their 20 s were selected as a sample, which was randomly and evenly divided into two

*Corresponding author. Young Mi Kim (E-mail: h1015m@naver.com)

(C2017 The Society of Physical Therapy Science. Published by IPEC Inc.

This is an open-access article distributed under the terms of the Creative Commons Attribution Non-Commercial No Derivatives (by-nc-nd) License $<$ http://creativecommons.org/licenses/by-nc-nd/4.0/>. 
groups: (1) the WT Group (with a TLSO) and (2) the WOT group (without a TLSO). The inclusion criteria included (1) no neurologic disease history, (2) capability to maintain independent standing, walking, and landing, (3) no surgical history that could affect walking or landing, and (3) no inborn deformity or disease in the relevant areas, including the foot, pelvis, and spine.

For the WT group, the average age was $24.9 \pm 2.4$ years, the average height was $173.3 \pm 4.2 \mathrm{~cm}$, and the average weight was $70.6 \pm 6.6 \mathrm{~kg}$. For the WOT group, the average age was $25.3 \pm 2.7$ years, the average height was $175.6 \pm 4.4 \mathrm{~cm}$, and the average weight was $69.1 \pm 6.8 \mathrm{~kg}$. Using Levene's variance homogeneity test and a normality test on the general physical characteristics, no statistically significant difference was found between the two groups $(p>0.05)$. All of the patients understood the purpose of this study and provided written informed consent prior to participation in accordance with the ethical principles of the Declaration of Helsinki.

The spinal orthosis used in this study was a TLSO called a plastic body jacket. It is made of lightweight polypropylene that has thermoplasticity, and is designed so that it is easy to put on and take off. It is a commercially available product that comes in four sizes (extra-large, large, medium, and small), and the appropriate size was used for the body figure of each research subject. More than any other TLSO, this particular plastic body jacket is known to be effective for maintaining the body's alignment and for effectively restricting movement in three 3-dimensional planes: (1) flexion and extension of the body's trunk in the sagittal plane, (2) lateral flexion in the frontal plane, and (3) rotation of the trunk of the body in the horizontal plane.

The steps of the staircase fabricated and used in this study were $17 \mathrm{~cm}$ high, $28 \mathrm{~cm}$ wide, $61 \mathrm{~cm}$ across, and were fabricated according to housing construction standards; four steps were installed for this study. A motion analysis system composed of six infrared cameras (Vicon motion capture system, Vicon Motion Systems Ltd., Oxford, UK) was used for measurement of the spatiotemporal variables and for the kinematic variables of walking, with the sample collection rate of the infrared camera set at $120 \mathrm{~Hz}$.

The subjects were told to walk comfortably and naturally down a 10-m long straight walking lane drawn on firm, even ground at their usual speed while looking straight ahead. Walking on level ground was performed after oral instruction, and this was followed by ascending and descending the stairs. The measurement interval was not mentioned so that the subjects were not aware of when the measurements began. Walking was measured after the first five footsteps, and a 30-second rest period was given after each motion.

SPSS for Windows 17.0 (IBM SPSS, Chicago, IL, USA) was used for the statistical data analysis. An independent t-test was used, given the homogenous nature of the subject; the difference WT and WOT group was calculated and comparisons were made using an independent t-test. The statistical significance level was set at $\alpha=0.05$.

\section{RESULTS}

Table 1 shows the angle values (mean and standard deviation) for the lower extremities at heel strike, midstance, toe off, and mid-swing in the sagittal plane. In the level ground walking measurements, significance differences were found at the $\mathrm{H} 2$ and $\mathrm{K} 2$ phases between the WT group and the WOT group $(\mathrm{p}<0.05)$. In the stair walking measurements, statistically significant differences were found at the $\mathrm{H} 2, \mathrm{H} 3$, and $\mathrm{K} 1$ phases during stair ascent and at the $\mathrm{H} 2, \mathrm{H} 3, \mathrm{~K} 2$, and $\mathrm{A} 1$ phases during stair descent between the two groups $(\mathrm{p}<0.05)$.

Table 1 compares the angle values (mean and standard deviation) for the lower extremities at heel strike, during stance, toe off, and during swing in the frontal plane between the WT group and the WOT group. In the level ground walking measurements, statistically significant differences were found at the H6 phase; in the stair walking measurements, significant differences were found at the $\mathrm{H} 5$ and $\mathrm{H} 7$ phases during ascent and at the K6 phase during descent ( $<0.05)$. Table 1 shows the angle values (mean and standard deviation) for the lower extremities at heel strike, during stance, toe off, and during swing in the horizontal plane between the WT group and the WOT group. The angle values for the hip joints, knee joints, and ankle joints between the two groups did not show significant differences in any of the walking phases in level ground walking ( $>0.05$ ), while a significant difference was found only at the K12 phase while descending the stairs.

\section{DISCUSSION}

When the kinematic variables in the lower extremities were compared in the sagittal plane, the flexion angles of the hip joints were considerably increased in $\mathrm{H} 2$ during both the level ground and the stair walking, an increase that seemed to compensate for restricted pelvic movements due to the spinal orthosis. Increase in the flexion angle at K1 and K2 and in the plantarflexion angle at A1 in the stair walking may serve as compensation for shock absorption against the ground reaction. A research by Konz reported that pelvic obliquity and pelvic rotation were remarkably reduced when a TLSO was applied and such pelvic reduction in the frontal and the horizontal planes affected the lower extremity movements during gait ${ }^{6}$. Another research reported that plantar flexion of the ankle joints in the loading response and knee joint flexion in the stance phase decreased the shock from the ground reaction ${ }^{7)}$. In this context, restriction in pelvic movements due to the spinal orthosis induced increased flexion in the ankle, knee, and hip joints in the sagittal plane in order to alleviate the vertical ground reaction. As for changes in the ankle joint angles in the sagittal plane in stair walking, dorsiflexion occurred between the initial contact 
Table 1. Comparison of joint angle between with TLSO group and without TISO group (unit: ${ }^{\circ}$ )

\begin{tabular}{|c|c|c|c|c|c|c|c|c|c|}
\hline \multirow{2}{*}{ Gait condition } & & \multicolumn{2}{|c|}{ Sagittal plane } & & \multicolumn{2}{|c|}{ Frontal plane } & & \multicolumn{2}{|c|}{ Transverse plane } \\
\hline & & without TLSO & with TLSO & & without TLSO & with TLSO & & without TLSO & with TLSO \\
\hline level walking & & $47.1 \pm 9.1$ & $51.9 \pm 5.9$ & & $-2.9 \pm 1.4$ & $-2 \pm 1.6$ & & $3.6 \pm 1.3$ & $3.4 \pm 1.1$ \\
\hline stair ascent & H1 & $64.9 \pm 10.2$ & $68.2 \pm 9.1$ & H5 & $4.9 \pm 1.6$ & $3.8 \pm 1.5^{*}$ & H9 & $7.3 \pm 1.7$ & $6.6 \pm 2.1$ \\
\hline stair descent & & $27.9 \pm 4.6$ & $30.2 \pm 4.3$ & & $-4.4 \pm 4$ & $-5.2 \pm 2.1$ & & $0.5 \pm 1.1$ & $0.9 \pm 1.9$ \\
\hline level walking & & $8.4 \pm 0.9$ & $14.9 \pm 3.8^{*}$ & & $2.3 \pm 0.9$ & $1.2 \pm 1^{*}$ & & $-6 \pm 4$ & $-4.2 \pm 2.8$ \\
\hline stair ascent & $\mathrm{H} 2$ & $10.5 \pm 1.8$ & $18.9 \pm 6.5^{*}$ & H6 & $3.3 \pm 1.4$ & $2.7 \pm 1.3$ & $\mathrm{H} 10$ & $-8.2 \pm 3.8$ & $-9.9 \pm 3$ \\
\hline stair descent & & $30.2 \pm 7.3$ & $35.4 \pm 5.9^{*}$ & & $4.6 \pm 1.5$ & $5.1 \pm 1.5$ & & $-4.2 \pm 3.8$ & $-3 \pm 2.3$ \\
\hline level walking & & $18.9 \pm 5.2$ & $17.7 \pm 5.1$ & & $-8.5 \pm 1.7$ & $-7.7 \pm 2.3$ & & $-3 \pm 1.7$ & $-2.2 \pm 2.8$ \\
\hline stair ascent & H3 & $10.9 \pm 2.5$ & $16.5 \pm 4^{*}$ & $\mathrm{H} 7$ & $-7.8 \pm 2.0$ & $-5.9 \pm 2.1$ & H11 & $-5.6 \pm 2$ & $-6.6 \pm 2.4$ \\
\hline stair descent & & $19.4 \pm 7.1$ & $30.9 \pm 6.1^{*}$ & & $-4.3 \pm 2.1$ & $-3.9 \pm 2$ & & $-5.6 \pm 2.6$ & $-5.7 \pm 3.1$ \\
\hline level walking & & $48.0 \pm 5.6$ & $48.3 \pm 5.9$ & & $-5.6 \pm 1.5$ & $-6.3 \pm 1.4$ & & $6.5 \pm 1.6$ & $6.4 \pm 1.7$ \\
\hline stair ascent & $\mathrm{H} 4$ & $17.7 \pm 2.9$ & $19.2 \pm 4.2$ & H8 & $-9.3 \pm 1.9$ & $-8 \pm 4.1$ & H12 & $7.5 \pm 1.4$ & $7.7 \pm 1.5$ \\
\hline stair descent & & $40.6 \pm 8.1$ & $42.2 \pm 9.4$ & & $-5.6 \pm 2.6$ & $-4.6 \pm 1.8$ & & $5.9 \pm 1.8$ & $5.6 \pm 1.6$ \\
\hline level walking & & $16.1 \pm 4.3$ & $19.2 \pm 5.4$ & & $2.4 \pm 1$ & $3 \pm 1.9$ & & $-8.4 \pm 1.8$ & $-7.9 \pm 2.2$ \\
\hline stair ascent & K1 & $60.8 \pm 9.1$ & $66.6 \pm 8.4^{*}$ & K5 & $5.7 \pm 1.9$ & $5.9 \pm 2.1$ & K9 & $-10.3 \pm 1.8$ & $-11.2 \pm 2.9$ \\
\hline stair descent & & $19.4 \pm 4.4$ & $22.4 \pm 5.2$ & & $4.2 \pm 2.4$ & $4.5 \pm 2.3$ & & $-6.2 \pm 1.9$ & $-8 \pm 3.9$ \\
\hline level walking & & $10.6 \pm 2.5$ & $15.8 \pm 3.4^{*}$ & & $5.2 \pm 1.7$ & $6.1 \pm 2.2$ & & $-7.7 \pm 1.8$ & $-7.3 \pm 2.1$ \\
\hline stair ascent & K2 & $20.9 \pm 4.7$ & $21.9 \pm 3.1$ & K6 & $6.9 \pm 1.8$ & $5.9 \pm 1.9$ & K10 & $-1.9 \pm 1.2$ & $-1.7 \pm 1.5$ \\
\hline stair descent & & $11.5 \pm 3.2$ & $17.7 \pm 3.5^{*}$ & & $7.3 \pm 2.3$ & $5 \pm 2 *$ & & $-5.1 \pm 2.2$ & $-5.5 \pm 2$ \\
\hline level walking & & $32.6 \pm 11.7$ & $33.4 \pm 8.2$ & & $-1.8 \pm 1$ & $-1.3 \pm 0.9$ & & $-3.1 \pm 1.8$ & $-3.2 \pm 1.9$ \\
\hline stair ascent & K3 & $28.7 \pm 6.9$ & $25.7 \pm 8.1$ & K7 & $-3.7 \pm 0.7$ & $-3.4 \pm 0.9$ & K11 & $-5.5 \pm 1.5$ & $-5.7 \pm 1.9$ \\
\hline stair descent & & $73.4 \pm 10.5$ & $71.2 \pm 22.6$ & & $-2.7 \pm 1.1$ & $-2.7 \pm 1.5$ & & $-8 \pm 2.8$ & $-9.1 \pm 2.4$ \\
\hline level walking & & $47.5 \pm 4.2$ & $46.9 \pm 5.1$ & & $-3.3 \pm 1$ & $-3.4 \pm 1.6$ & & $0.6 \pm 1.9$ & $-0.1 \pm 1.1$ \\
\hline stair ascent & K4 & $81.2 \pm 12.3$ & $81.1 \pm 11.4$ & K8 & $-4.3 \pm 1$ & $-4.4 \pm 1.5$ & K12 & $15.8 \pm 6.4$ & $13.9 \pm 3.1$ \\
\hline stair descent & & $84.8 \pm 9.2$ & $79.6 \pm 11.2$ & & $-4.3 \pm 1.3$ & $-4.2 \pm 1.1$ & & $6.2 \pm 2$ & $9.2 \pm 3.8^{*}$ \\
\hline level walking & & $3.4 \pm 1.9$ & $2.7 \pm 1.2$ & & $3.9 \pm 1.6$ & $3.1 \pm 0.9$ & & $-12.6 \pm 4.4$ & $-12.4 \pm 3.5$ \\
\hline stair ascent & A1 & $14.3 \pm 3.9$ & $12.7 \pm 3.9$ & A5 & $6.4 \pm 1.4$ & $5.6 \pm 1.5$ & A9 & $-27.5 \pm 3.9$ & $-26.6 \pm 6.2$ \\
\hline stair descent & & $-16.0 \pm 5.6$ & $-21.2 \pm 4.9$ & & $3.3 \pm 1.2$ & $3.7 \pm 1.3$ & & $-13 \pm 4.6$ & $-13.6 \pm 7.3$ \\
\hline level walking & & $5.8 \pm 1.4$ & $5.7 \pm 1.1$ & & $4.7 \pm 2.1$ & $5.4 \pm 1.5$ & & $-11.8 \pm 3.6$ & $-10.5 \pm 2.9$ \\
\hline stair ascent & A2 & $6.6 \pm 3.2$ & $6.6 \pm 3.6$ & A6 & $5.2 \pm 1.7$ & $4.3 \pm 1.3$ & A10 & $-20.9 \pm 7.5$ & $-17.9 \pm 4.3$ \\
\hline stair descent & & $15.3 \pm 5.6$ & $13.8 \pm 4.1$ & & $3.7 \pm 1.4$ & $3.9 \pm 2.2$ & & $-14.9 \pm 5$ & $-17.5 \pm 4.2$ \\
\hline level walking & & $-11.8 \pm 2.8$ & $-13.6 \pm 3.7$ & & $3.6 \pm 1.4$ & $3.3 \pm 1$ & & $-9.1 \pm 2.2$ & $-8.9 \pm 2.4$ \\
\hline stair ascent & A3 & $-9.4 \pm 1.9$ & $-10.4 \pm 2.6$ & A7 & $4.6 \pm 1.5$ & $5.4 \pm 2.3$ & A11 & $-7.6 \pm 2.7$ & $-6.7 \pm 2$ \\
\hline stair descent & & $2.3 \pm 1.3$ & $2.3 \pm 1.1$ & & $4.1 \pm 1.8$ & $4.3 \pm 2$ & & $-13.8 \pm 4.8$ & $-16.3 \pm 5.2$ \\
\hline level walking & & $3.8 \pm 1.4$ & $3.8 \pm 0.6$ & & $6.2 \pm 1.9$ & $5.5 \pm 1.4$ & & $-24.4 \pm 5.3$ & $-22.2 \pm 12.2$ \\
\hline stair ascent & A4 & $14.5 \pm 19.9$ & $10.2 \pm 5.8$ & A8 & $7.1 \pm 1.8$ & $6.9 \pm 1.9$ & $\mathrm{~A} 12$ & $-31.1 \pm 5.2$ & $-31.7 \pm 4.9$ \\
\hline stair descent & & $-12.4 \pm 3.5$ & $-11.8 \pm 3.1$ & & $6.6 \pm 1.9$ & $6.4 \pm 2.2$ & & $-25.4 \pm 6.8$ & $-28.5 \pm 5.8$ \\
\hline
\end{tabular}

*Significantly different between with TLSO and without TLSO $(\mathrm{p}<0.05)$, TLSO: Thoracolumbosacral orthosis

H1: Hip flexion/extension at initial contact, H2: Hip maximum flexion/extension in midstance phase, H3: Hip flexion/extension at toe off, H4: Hip maximum flexion/extension in midswing phase, K1: Knee flexion/extension at heel strike, K2: Knee maximum flexion/ extension in midstance phase, K3: Knee flexion/extension at toe off, K4: Knee maximum flexion/extension in midswing phase, A1: Ankle dorsflexion/plantarflexion at heel strike, A2: Ankle maximum dorsiflexion/plantarflexion in midstance phase, A3: Ankle dorsiflexion/Plantarflexion at toe off, A4: Ankle maximum dorsiflexion/plantarflexion in midswing phase, H5: Hip adduction/abduction at heel strike, H6: Hip maximum adduction/abduction in midstance phase, H7: Hip adduction/abduction at toe off, H8: Hip maximum adduction/abduction in midswing phase, K5: Knee varus/valgus at heel strike, K6: Knee maximum varus/valgus in midstance phase, K7: Knee varus/valgus at toe off, K8: Knee maximum varus/valgus in midswing phase, A5: Ankle inversion/eversion at heel strike, A6: Ankle maximum inversion/eversion in midstance phase, A7: Ankle inversion/eversion at toe off, A8: Ankle maximum inversion/eversion in midswing phase, H9: Hip int/external rotation at heel strike, H10: Hip maximum int/external rotation in midstance phase, H11: Hip int/external rotation at toe off, H12: Hip maximum int/external rotation in midswing phase, K9: Knee int/external rotation at heel strike, K10: Knee maximum int/external rotation in midstance phase, K11: Knee int/external rotation at toe off, K12: Knee maximum int/external rotation in midswing phase, A9: Ankle int/external rotation at heel strike, A10: Ankle maximum int/external rotation in midstance phase, A11: Ankle int/external rotation at toe off, A12: Ankle maximum int/external rotation in midswing phase 
and the initial phase of ascent, followed by gradual plantar flexion and then sudden plantar flexion at the middle phase, while maximal plantar flexion was shown at initial descent, followed by rapid dorsiflexion until the early part of the gaiting phase and then gradual dorsiflexion during the rest of the phase, a change that seemed to absorb the shock of the body load ${ }^{8)}$.

Changes in the angles of the lower extremities in the sagittal plane in this study showed significant differences in stair gaiting rather than in level ground walking; thus, the WT group showed increased flexion in their lower extremities.

In the angles of the lower extremity motions in the frontal plane, the internal rotation was reduced at H6 during level ground walking, at $\mathrm{H} 5$, and at $\mathrm{H} 7$ during stair ascent, and at $\mathrm{K} 6$ during stair descent, a reduction that may be consistent with the results of the research by Konz, which reported that changes in the motion angles in the thigh and the pelvic segment in the frontal plane due to spinal orthosis application were directly associated with reduction in the pelvic oblique.

In the angles of the lower extremity motions in the horizontal plane, the internal rotation was increased at K12 only during stair descent, an increase that is consistent with the results of the research by Konz, which reported that no differences were found in the angles of the lower extremity motions in the horizontal plane during level ground walking.

In the present study, significant differences in the sagittal plane were shown in stair walking rather than in level ground walking. The increase in flexion in the lower extremities might be caused by an increase in the knee joint flexion and the plantar flexion of the ankle joints to compensate for reduced shock absorption against the ground reaction due to the restriction in trunk movements caused by the application of the spinal orthosis. It can be concluded that a spinal orthosis is useful in stabilizing the lower extremities during stair gaiting, and that appropriate application of the orthosis plays a supporting role in the activities of daily life and therapeutic intervention.

\section{REFERENCES}

1) Pinto RZ, Souza TR, Trede RG, et al.: Bilateral and unilateral increases in calcaneal eversion affect pelvic alignment in standing position. Man Ther, 2008, 13: 513-519. [Medline] [CrossRef]

2) Khamis S, Yizhar Z: Effect of feet hyperpronation on pelvic alignment in a standing position. Gait Posture, 2007, 25: 127-134. [Medline] [CrossRef]

3) Haight HJ, Dahm DL, Smith J, et al.: Measuring standing hindfoot alignment: reliability of goniometric and visual measurements. Arch Phys Med Rehabil, 2005, 86: 571-575. [Medline] [CrossRef]

4) Duval K, Lam T, Sanderson D: The mechanical relationship between the rearfoot, pelvis and low-back. Gait Posture, 2010, 32: 637-640. [Medline] [CrossRef]

5) Weinstein SL, Dolan LA, Wright JG, et al.: Effects of bracing in adolescents with idiopathic scoliosis. N Engl J Med, 2013, 369: 1512-1521. [Medline] [CrossRef]

6) Konz R, Fatone S, Gard S: Effect of restricted spinal motion on gait. J Rehabil Res Dev, 2006, 43: 161-170. [Medline] [CrossRef]

7) Gard SA, Childress DS: The influence of stance-phase knee flexion on the vertical displacement of the trunk during normal walking. Arch Phys Med Rehabil, 1999, 80: 26-32. [Medline] [CrossRef]

8) Kim YS, Seo CJ: The comparative analysis of EMG and gait patterns of lower extremities during going up stairs and down. Kor J Phys Edu, 2006 , 45: 535-545. 